\title{
Competencias formativas delos investigadores noveles según los investigadores consolidados
}

TOMAS FONTAINES-RUIZ

Universidad Técnica de Machala - Ecuador

\author{
ARMANDO ISAIAS CARHUACHÍN MARCELO* \\ Universidad Nacional Daniel Alcides Carrión - Cerro de Pasco - Perú
}

FLAVIANO ARMANDO ZENTENO RUIZ ${ }^{* *}$

Universidad Nacional Daniel Alcides Carrión - Cerro de Pasco - Perú

FERNANDA TUSA JUMBO****

Universidad Técnica de Machala - Ecuador

Recibido el 01-05-2017; primera evaluación el 11-06-2018; segunda evaluación el 26-06-2018; aceptado el 29-06-2018

\footnotetext{
" Psicopedagogo (Summa Cum Laude), licenciado en Educación, mención: Ciencias Pedagógicas, área: Orientación (Summa cum Laude); magíster en Orientación Educativa; especialista en metodología de la investigación; doctor en Ciencias Humanas; postdoctorado en métodos, técnicas y metodologías aplicadas a las ciencias sociales y humanidades; postdoctorado en procesos sincrónicos y diacrónicos de la ciencia; doctorando en Estudios del Discurso. Director de Investigación de la Universidad Técnica de Machala, Ecuador. Exbecario del programa Prometeo-Ecuador. Correo: tfontaines@hotmail.com

" Doctor en Ciencias de la Educación con maestría en Educación Matemática. Licenciado en Educación Matemática-Biología. Formador en matemática. Coordinador académico del programa de Formación y Capacitación Permanente. Docente capacitador en Didáctica y Gestión Pedagógica de la Educación. Coordinador general de capacitación de profesores y directores. Director de la Escuela de Profesionalización Docente, director de pregrado y posgrado en Unidades de Educación. Correo: armandois61@hotmail.com

${ }^{* * *}$ Doctor en Ciencias de la Educación. Máster en Educación Matemática con varios diplomados y especializaciones en educación, currículo y estrategias didácticas. Especialista en Programa de Formación en Formulación de Proyectos de Inversión Pública. Consultor de empresas educativas. Docente en la Universidad Nacional Daniel Alcides Carrión y docente, a nivel de posgrado, en importantes universidades públicas y particulares del país. Correo: armandozenteno77@gmail.com

**** Comunicadora social. Doctora en Comunicación Social de la Universidad Austral de Argentina. Máster Universitario en Medios en Red y Ciencias de la Web (Universidad de Alcalá). Máster Internacional en Comunicación y Educación. Docente de la carrera de Comunicación Social, adscrita a la Unidad Académica de Ciencias Sociales de la Universidad Técnica de Machala, Ecuador. Autora de varios libros, capítulos de libro y artículos científicos en la línea de investigación «Comunicación y Literatura».Correo: ftusa@utmachala.edu.ec
} 


\section{Resumen}

El objetivo del estudio fue develar las representaciones que tienen los investigadores consolidados sobre las competencias formativas de los investigadores noveles. El estudio fue descriptivo con un diseño mixto. Los datos derivan de un corpus textual construido a partir de las opiniones de 78 investigadores Prometeos sobre los conocimientos y habilidades que deben tener los investigadores noveles. Los resultados revelaron dos macro núcleos de competencias: las prosociales y emocionales; y las metodológicas-discursivas-tecnológicas. Se resaltó el carácter socioemocional de la investigación como objeto de formación y la necesidad de liberar las prácticas metodológicas del dogmatismo y las pasiones de los formadores. El novel investigador debe tener conciencia emocional, apertura hacia la pluralidad metódica, habilidades para utilizar eficientemente la tecnología en favor de su estudio y calidad discursiva para la socialización de sus saberes.

Palabras clave: formación de investigadores, investigadores, competencias investigadoras, discursos

\section{Training competences of novice researchers from strengthen researcher's views}

\section{Abstract}

The purpose of this study was to reveal the conceptions that expert researchers have about novel researcher's competences. The study was descriptive, with a mixed design. The data comes from a textual corpus constructed from 78 Prometeo researchers' opinions about the type of knowledge and skills that novel researchers need. The results revealed two competences cores: the prosocial-emotional and methodological-discursive-technological competences. The expert researchers emphasized the socio-emotional character of research as the need to release the methodological practices from the dogmatism and the passions of the instructors. Novel researchers must have emotional consciousness, openness to methodical plurality, ability to use efficiently technology in favor to their research studies, and discursive quality for the socialization of knowledge.

Keywords: researchers training, researchers, research competencies, discourse

\section{Competências formativas de jovens pesquisadores segundo pesquisadores experientes}

\section{Resumo}

O objetivo do estudo foi identificar as representações que possuem os pesquisadores Júnior sobre as competências de formação dos investigadores Jovens. O estudo foi descritivo com um desenho misto. Os dados foram obtidos de um corpus textual construído a partir das opiniôes de 78 pesquisadores Sênior sobre os 
conhecimentos e habilidades que devem ter os pesquisadores Jovens. Os resultados revelaram dois macronúcleos de competição alvo: as pró-sociais e emocionais; e as metodológicas-discursivas-tecnológicas. Foi realçado o caracter socio-emocional da pesquisa e a necessidade de liberar as práticas metodológicas do dogmatismo e as paixóes dos formadores. O investigador jovens deve ter consciência emocional, abertura às pluralidades metódicas, habilidades para o uso eficiente da tecnologia para o seu estudo e qualidade discursiva para a socializaçáo de seus conhecimentos.

Palavras chave: formação de pesquisadores, pesquisadores, competências pesquisadores, discursos

\section{INTRODUCCIÓN}

La formación de investigadores, en la actualidad, es aprehendida desde diversas perspectivas. Ha sido abordada como: a) un proceso interdisciplinario y de búsqueda o contacto con la información (Rodriguez, Alvite y Olea, 2015; Salgado, Ortiz y Rogel, 2016); b) la consecuencia del ejercicio didáctico-tutorial (Dietz, 2014; Torrecilla, Cartagena, Crespo, Oyarzabal y Pérez, 2017); c) el producto de las políticas públicas (Rivas, 2004); d) una disposición organizacional (Gotuzzo, Gonzalez y Verdonck, 2010); e) el resultado de disposiciones personales (Giménez, Pedrazas, Medina y Dalmau, 2009; Moreno-Casbas, Fuentelsaz-Gallego, González-María y Gil de Miguel, 2010). Sin embargo, su comprensión como práctica social y sociodiscursiva aún está pendiente. De allí que nos planteamos, como objetivo principal, develar las representaciones que tienen los investigadores consolidados sobre las competencias que deben considerarse para impulsar la formación de investigadores noveles.

Partiendo de ello, el presente estudio concibe al investigador experto como informante clave en la construcción del investigador novel. Suponemos que quien investiga se adapta y responde a las complejas demandas del entorno, en una sociedad que se caduca al ritmo del reloj y, al hacerlo, configura la investigación como práctica social, cuyos sentidos están condicionados por la interacción entre su marco cognitivo, la comunidad epistémica a la que pertenece y las variaciones implícitas en su objeto de estudio y perspectivas de abordaje.

En atención a estos cambios, precisamos el modo en que la comunidad científica concibe la preparación de las generaciones de relevo frente a los entornos complejos. La meta fue irrumpir en la cognición social de la formación investigadora y exponer cuáles son los componentes más urgentes para formar al investigador novel, partiendo de la experiencia cotidiana de quienes están en los equipos de investigación. Asimismo, se ponen al descubierto rasgos 
de personalidad que garantizan la participación y sostenibilidad de los noveles investigadores en los espacios de aprendizaje. Estas claves servirán prospectivamente para diseñar programas de formación institucional que posicionen investigadores capaces de adaptarse y desarrollar procesos de reingeniería personal y social mediante la comprensión de los dispositivos discursivos que condicionan su desempeño.

\section{MarCo teórico}

\subsection{Representaciones sociales}

Las representaciones sociales fusionan aportes derivados de la psicología de los pueblos (Wundt, 1926), el interaccionismo simbólico (Blumer, 1969) y las representaciones colectivas (Durkheim, 2000), respecto a la legitimidad del conocimiento ingenuo y la interacción como fuentes explicativas de la relación entre individuos y sus modos de significación de la realidad (Mora, 2002). Su creador fue Moscovici (1979) quien las concibe como entidades hechas de sustancia simbólica, que son casi tangibles, se cruzan en nuestra cotidianidad y son las responsables de preparar al individuo para la acción y de generar las condiciones contextuales para que se produzcan.

La versatilidad de este concepto ha dado lugar a distintas miradas ontológicas, epistemológicas y metodológicas. Pasamos de la aprehensión hermenéutica del objeto (Moscovici, 1979; Jodelet, 2007) a la identificación de un núcleo estable de sentido que regula el proceso de significación y actuación diferencial de los grupos sociales que comparten esa representación (Abric, 2001; Flament, 2001). Pero también dejamos constar una especie de interdependencia entre la representación y el individuo, quien tiene la posibilidad de juzgar su adherencia a la misma, de acuerdo a su voluntad o el nivel de afectación que esta le genere (Doise, Spini y Clémence, 1999). En la actualidad, el estudio de las representaciones sociales ha migrado de la psicología hacia el campo de la salud (Viard et al., 2016), educación (NetzahualcoyotiNetzahual, 2015), ambiente (Diaz y Gómez, 2005), política, entre otros (Gutiérrez y Campos, 2010; Gutiérrez y Cuevas, 2012).

Entre las características más resaltantes de este constructo, rescatamos las siguientes: a) están aferradas a la cultura y por lo tanto, son referentes para condicionar la memoria social y el modo en que interactuamos y construimos sentidos psicoafectivos a partir de nuestras experiencias (Banchs, 2000, 2001, 2007); b) le otorgan rango de verdad a la información que manejamos; c) ofrecen una estructura normativa para la interacción humana alrededor del 
fenómeno representado; d) son mediadoras de la interacción comunicativa y corresponsables de la concepción de realidad que tiene el sujeto; e) son casi tangibles y viajan a través del discurso para darle sentido a las prácticas sociales (Fairclough, 2008, 2012), de allí que el modo en que estructuramos la realidad sea una infinita semiosis que revela la posición del sujeto en relación con la práctica social en la que actúa.

En cuanto a la formación de las representaciones, Moscovici (1979) indica que se deben vivir dos procesos: la objetivación y el anclaje. La objetivación ocurre cuando se identifica el núcleo que encierra el sentido de la representación y, a partir de él, se traman redes multicausales para poder explicar, clasificar, juzgar y tomar decisiones sobre lo que sucede en el mundo material. A partir del uso cotidiano de estos contenidos, se activa el proceso de anclaje de la representación en la cognición del sujeto, hecho que se hace palmario durante la toma de decisiones, la interpretación de signos y símbolos que hacen posible la interacción comunicativa.

Las representaciones están llamadas a ser una epistemología del conocimiento cotidiano, donde los saberes sociales son la base para representar los saberes científicos y, considerando que ambos viajan a través del discurso, están sometidas a constantes conflictos, significaciones y re-significaciones que, en definitiva, terminan siendo un reflejo del modo en que se organiza la realidad desde una perspectiva construccionista. En esta investigación, reconocemos que las representaciones se construyen y significan dentro de una comunidad, mediante la interacción sociodiscursiva. Identificamos que toda representación presenta un núcleo-ideología, que regula el discernimiento entre lo cierto e incierto, al estilo de un panóctico social (Foucault, 2005), donde se castiga todo aquello que se opone a la verdad establecida y se refuerza el comportamiento estandarizado, de allí que indaguemos en el discurso de los investigadores consolidados los sentidos de la formación de investigadores, teniendo como unidad de análisis sus competencias.

\subsection{Sobre las competencias como categoría discursiva}

Las investigaciones de McClelland (1973) impulsan el estudio de las competencias como medio para la selección del personal en la empresa. La mutabilidad de este concepto diversificó su uso y sentidos teóricos, al punto que, en la actualidad, se considere un término polisémico y difícil de aprehender en su totalidad. En esta investigación, no pretendemos teorizar sobre los textos seminales de este constructo; contrario a ello, lo empleamos como una macrocategoría discursiva para ordenar los saberes, habilidades y destrezas 
que debe poseer el investigador novel, según el investigador experto. La razón de esta selección obedece a que, en la formación del investigador, se reclaman actitudes, habilidades y conocimientos que tienen alto nivel de convergencia y que son orquestados desde una ideología que viabiliza el curso de la práctica científica.

Las competencias han sido tratadas como: capacidades para desarrollar una actividad determinada (Chaui, 2006); procesos complejos de desempeño con idoneidad contextual y responsabilidad (Tobon, Rial Sanchez, Carretero y Garcia, 2006); la puesta en escena de comportamientos que sintetizan rasgos de personalidad, aptitudes y conocimientos (Lévy-Leboyer, 1997). También son vistas como la capacidad de movilizar conocimientos, habilidades y actitudes para realizar actividades con calidad y eficacia (Bisquerra y Pérez, 2007; Bisquerra, 2005). Las competencias implican entrecruzamiento de capacidades, habilidades y actitudes, para garantizar un desempeño coherente con las demandas del contexto, pero también permiten inferir que existe un elemento oculto que condiciona su idoneidad en relación con las tareas y contextos de aplicación. Esto pone en evidencia la episódica dominancia de un paradigma que termina por convertir al sujeto en objeto social y luego lo reifica (Chaui, 2006). Suponemos que la selección de las competencias están orquestadas por ideologías que circulan en el discurso como una especie de dispositivos y que terminan por decantar a los sujetos que tienen o no el perfil para gestionar ciertas actividades (Foucault, 2001).

Si trasladamos estas ideas a la formación de investigadores, supondríamos que las instituciones han definido un conjunto de condiciones cognitivas, procedimentales y afectivas que debe tener un sujeto para ser investigador $y$ tener el reconocimiento de la comunidad científica. Las instituciones avalan un discurso competente que es aceptado y escuchado como verdadero (Chaui, 2006) y lo instituyen a través del currículo, para disimular la imposición de una norma que regule el reconocimiento de la verdad. Es, en este punto, donde cobra sentido la resistencia de paradigmas o la convergencia entre ellos, respecto a qué debe tener el investigador, cómo debe proceder, cuáles son los modos más o menos efectivos de abordar y entender el objeto de estudio y, por supuesto, cómo generar respuestas pertinentes a las demandas de los tiempos de hoy.

Lo indicado revela que las competencias no pueden ser vistas como normas universales (Gimeno, 2008), aunque muchas de ellas se encuentren de manera recurrente en múltiples contextos disciplinarios y laborales. La moraleja es que, al viajar en el discurso, se hacen transindividuales y transfronterizas; por ello, es importante precisar su existencia para impulsar la conquista del saber metódicamente regulado. 


\section{Metodología de la inVestigación}

El estudio, de acuerdo a su objetivo, se consideró descriptivo con un diseño mixto (Fontaines-Ruiz, 2012; Creswell y Zhang, 2009; Creswell, 2014). Se trabajó con 78 investigadores miembros del Programa Prometeo de la Secretaría Nacional de Educación Superior, Ciencia y Tecnología de la República del Ecuador (SENESCYT) que aceptaron voluntariamente participar como informantes clave, en respuesta a la convocatoria interna ad hoc que impulsó la SENESCYT. Los Investigadores Prometeos, considerados en este estudio como investigadores consolidados, fueron seleccionados mediante muestreo opinático-intencional (Fontaines-Ruiz, 2012; Bonilla-Castro y Rodríguez, 2005). Entre sus características, están las siguientes: a) Eran nativos de Israel, España, Venezuela, Estados Unidos, Francia, Australia, Argentina, Portugal, Italia, Reino Unido, Cuba, México. b) El tiempo promedio de experiencia acumulada en la investigación fue de 15 años. c) Entre sus áreas de investigación están: las ciencias de la vida, agricultura, educación, ciencias sociales, ingeniería e industria, humanidades y artes. d) Acreditan, en su gestión académica, publicaciones científicas, tutorías de tesis de maestría y doctorado, gestión de proyectos de investigación, integración en redes internacionales en producción de conocimientos y obtención de premios como reconocimiento a su labor investigativa.

La unidad de análisis en esta investigación fue un corpus creado intencionalmente, a partir de las respuestas que dieron los informantes al cuestionario web, contenido de las siguientes interrogantes: a) ¿Cuáles son los conocimientos que debe tener el novel investigador para consolidar su formación?; b) ¿Cuáles son las habilidades que debe consolidar el investigador novel? La aplicación del mismo se hizo bajo la gestión del programa Prometeo. El análisis del corpus se realizó en las fases siguientes:

\section{Fase 1: Reducción del corpus}

La intención fue agrupar las palabras que tuviesen mayor energía textual, a fin de formar grupos u objetos jerárquicos que determinarán la tendencia semántica del texto objeto de análisis (Torres-Moreno, Molina, y Sierra, 2010). En esta fase se aplica el análisis estadístico de datos textuales (Bécue, Lebart, y Rajadell, 1992; Lebart y Salem, 1994). Se produce la construcción de un biplot que reporta el porcentaje de variabilidad explicada en el discurso analizado. Asimismo, se identifican los potenciales contextos lingüísticos a los que aluden los hablantes durante su interacción y se identifican los términos que revelan las características de personalidad de los investigadores. En estos análisis se empleó el software t-lab en español, versión 6.1 (Lancia, 2004). Este 
programa establece diferencias entre núcleos textuales, que son la base para la posterior organización temática de la representación.

\section{Fase 2. Estructuración de las representaciones}

Durante esta fase se construyeron las categorías discursivas en atención al nivel de cercanía semántica y el nivel de abstracción mostrado. Las categorías se fueron sincronizando de acuerdo a su nivel de complejidad, teniendo como esquema de referencia los planteamientos de Lakatos (2011) y Abric (2001). En tal sentido, se construyó el núcleo fuerte de la representación, el cual fue bordeado por un cinturón protector compuesto por artefactos discursivos potencialmente cambiables de acuerdo a la variabilidad del contexto.

\section{Fase 3. Interpretación de las representaciones}

Este momento de la investigación permitió la argumentación del modo en que funcionan las representaciones y su materialización en los dispositivos de formación de investigadores.

\section{Resultados}

Se identificaron cinco clúster que explican el 65,86\% de la varianza en el discurso de los investigadores consolidados sobre las competencias a formar en el novel investigador (ver figura 1). A partir de su composición semántica, los clúster recibieron la siguiente denominación: competencias sociales, emocionales, discursivas, metodológicas y tecnológicas. De manera intencional, y de acuerdo a la cercanía semántico-pragmática de los clúster, se identificaron dos macronúcleos interdependientes, los cuales asumiremos como los ejes cardinales del proceso de formación y a partir de los cuales se desglosan las competencias específicas que deberán ser atendidas. Estos macro-núcleos son: 1) competencias pro-sociales y emocionales del sujeto como condicionante de su ajuste intra e interpersonal; 2) competencias metodológicas-discursiva-tecnológicas. Esta composición refleja signos de cambio al interior de la comunidad científica, al ablandar sus concepciones sobre el sujeto que investiga y al hacer de la vida en redes, la cotidianidad de la dinámica institucional. Los clúster obtenidos presentaron indicadores de calidad de acuerdo con los valores siguientes: Calinski-Harabasz =8,143; Davies-Bouldin=2,689; ICC (rho)= 0,092.

$\mathrm{El}$ investigador experto, a través de su discurso, muestra que comunicar la ciencia, convivir con el otro, romper las barreras del idioma, democratizar lo que se conoce y cómo se conoce, interactuar en entornos físicos y virtuales, es parte de su identidad en los tiempos actuales (Tonso, 2006). Ya los conceptos 


\subsection{Competencias identificadas}

\subsubsection{Competencias identificadas}

Iniciaremos definiendo la competencia socioemocional como la habilidad para comprender las tensiones del entorno heurístico y social y responder de modo proactivo ante los requerimientos que de ellos se deriven. El discurso de los investigadores permitió identificar cuatro rasgos distintivos de esta competencia, a saber: la actitud emprendedora, el trabajo colaborativo, el afrontamiento de la adversidad y la apertura a la experiencia (ver figura 2). Estas disposiciones humanas las asumimos como competencias transversales vinculadas con el saber ser y el saber convivir.

La relevancia de esta dimensión es que impulsa la actitud positiva y de aceptación hacia la investigación y sus actividades conexas. También pone de relieve que la conciencia emocional protege al individuo de síntomas vinculados con el bournot y la pérdida de engagement, e influye en la formación de actitudes de resistencia o aceptación hacia las actividades de investigación (Cejudo y López-Delgado, 2017; Caro y Sicilia, 2014; Oriol-Granado, Mendoza-Lira, Covarrubias-Apablaza, y Molina-López, 2017). Aunado a lo señalado, las emociones son consideradas como inhibidores conductuales; incluso, llegan a incidir en el modo en que juzgamos la realidad que vivimos, de allí la necesidad de impulsar un proceso de gestión de las mismas, para garantizar un mayor ajuste al contexto de acción del investigador (Martínez-Lorca, Zabala-Baños y Aguado Romo, 2017; Rico, Alzate y Sabucedo, 2017; Zhang, Kong y Li, 2017)

Seguidamente están las competencias discursivas, las cuales concebimos como el conocimiento y habilidad para seleccionar y operar el género discursivo que mejor se ajuste a su necesidad comunicativa. En la actualidad, es imperativo que el investigador se alfabetice en materia de discurso académico y sus géneros constitutivos (artículos, ensayos, monografías, entre otros), con la finalidad de ganar pericia para comunicarse con sus pares. Es necesario destacar la necesidad de fomentar las competencias comunicativas en inglés ya que es la lengua en la que se publican las revistas de corriente principal.

El investigador novel debe tener la capacidad de comprender cómo se crean, mantienen y reproducen los saberes a partir de las interacciones discursivas, lo que exige la apropiación de símbolos y relaciones de resistencia y dominación en el interior de las comunidades epistémicas y de práctica donde se desarrollan. Esto supone que la identidad académica se construye discursivamente y se materializa en un conjunto de géneros y subgéneros que caracterizan la comunicación del saber científico y las formas de interacción social. Aunado a ello, se ha demostrado que la comprensión de los géneros discursivos facilita 
el aprendizaje de su estructura, y el modo en que el investigador se representa e interactúa con los textos, durante la preparación de manuscritos académicos (Bayón, 2015; Kostrova y Kulinich, 2015; Parkinson, Demecheleer y Mackay, 2017; Zare y Keivanloo-Shahrestanaki, 2017).

La competencia metodológica es la capacidad para aplicar de forma pura o híbrida los principios del método en correspondencia con la singularidad del proceso de investigación que se planifica o desarrolla. Los investigadores consolidados hacen particular hincapié en el conocimiento de variadas metodologías y formas de recolectar datos. Rompen con la linealidad metodológica ante las plurales percepciones vinculadas a su objeto en estudio (FontainesRuiz y Jiménez, 2016). Sin declararlo, otorgan vigencia a la invitación de Feyerabend $(1987,2008)$ sobre la necesidad de probar diversos modos de acercarnos al objeto, sin perder la prudencia metódica. La idea es identificar los requerimientos a investigar y poder diseñar respuestas potencialmente adaptables a la naturaleza de lo investigado, tal como se realiza desde las investigaciones que emplean métodos mixtos en sus abordajes (Criollo, Romero y Fontaines-Ruiz, 2017; Fontaines-Ruiz, 2012).

Vinculada a esta competencia está la necesidad de formar la habilidad para ubicar información en las bases de datos informatizadas. Hoy es impensable investigar sin mirar lo que se ha publicado y, en esta tarea, los repositorios y bases de datos son piezas clave para encontrar información actualizada sobre el nivel de profundidad que ha tenido el objeto de estudio y sus modos de abordaje, con la finalidad de gestionar investigaciones con mayor probabilidad de impacto e innovación en el contexto de aplicación. Como elemento de cierre, en estas competencias está la habilidad de gestionar procesos de investigación, como consecuencia de mirar el conocimiento a través de la transferencia, el desarrollo y fomento de la cultura de innovación.

La última de las competencias identificadas es la tecnológica. Los investigadores consolidados crearon un contexto metodológico para ubicar esta competencia. Se define como el conocimiento y habilidad para emplear softwares específicos para el análisis de datos, y el aprovechamiento de la web 2.0 para facilitar la gestión, difusión y divulgación de los productos académicos generados. La versatilidad de esta competencia está en el fomento del trabajo colaborativo, transindividual y transhistórico, entre colegas, facilitando la interacción y la posibilidad de lograr mayor visibilidad de los productos de investigación que se generan (ver figura 2). Hoy la investigación que no se socializa es letra muerta y, en este sentido, el manejo de la tecnología es clave para mantener al novel investigador en sintonía con las tendencias y tensiones de su área de conocimiento. 


\subsubsection{Re-construcción discursiva del novel investigador}

La construcción de estas representaciones ha dado lugar a la reconfiguración del investigador novel como sujeto. Si cambia el escenario de formación, también es necesario que se produzcan cambios en la actitud del sujeto ante el conocimiento y la investigación; por ello, se consideró necesario mirar los rasgos de personalidad, a partir de los adjetivos calificativos que emplearon los investigadores consolidados al referirse a los noveles (ver figura 3).

En correspondencia con lo señalado, se formaron cuatro categorías, atendiendo a su congruencia semántica. El primer grupo manifiesta el gozo del investigador en su práctica, representado en su pasión, creatividad, audacia, deseo y curiosidad, frente al proceso que desarrolla, que es comúnmente encapsulado bajo la noción de vocación (Jiménez y Duarte, 2013). Por no haber investigación sin alegría, la ardua tarea de buscar «agujas en pajares» reclama una actitud resiliente y competitiva. Hace falta capital psíquico para afrontar los obstáculos inherentes a la tarea de investigar. También es necesario alimentar la voluntad para mantener la estrategia de búsqueda de los insumos para la satisfacción de sus requerimientos. Los investigadores consolidados representan la resiliencia a través de la tenacidad, optimismo, espíritu de sacrificio, perseverancia y ambición que debe tener el novel investigador para no abandonar su práctica y responder ante las demandas que realizan los formadores y la comunidad científica en general para reconocer la verdad de los hallazgos que se están reportando.

Otra de las categorías están ligadas a las actitudes clásicas del científico, asociadas a la rigurosidad y sistematicidad, lo cual forma parte del legado del método científico y la actitud heredada del ethos mertoniano (Merton, 1977). Esta categoría hace constar que la emocionalidad es compatible con la rigurosidad, y profundidad del pensamiento como muestra de la inalterable postura del investigador ante su objeto y que, además, hay coexistencia entre nuestra subjetividad al vivir la investigación y la ecuanimidad, crítica y rigor en su reporte. La moraleja es que contactar con la alegría de investigar no invalida lo que hacemos, contrario a ello, lo humaniza y hace significativo y trascendente. 


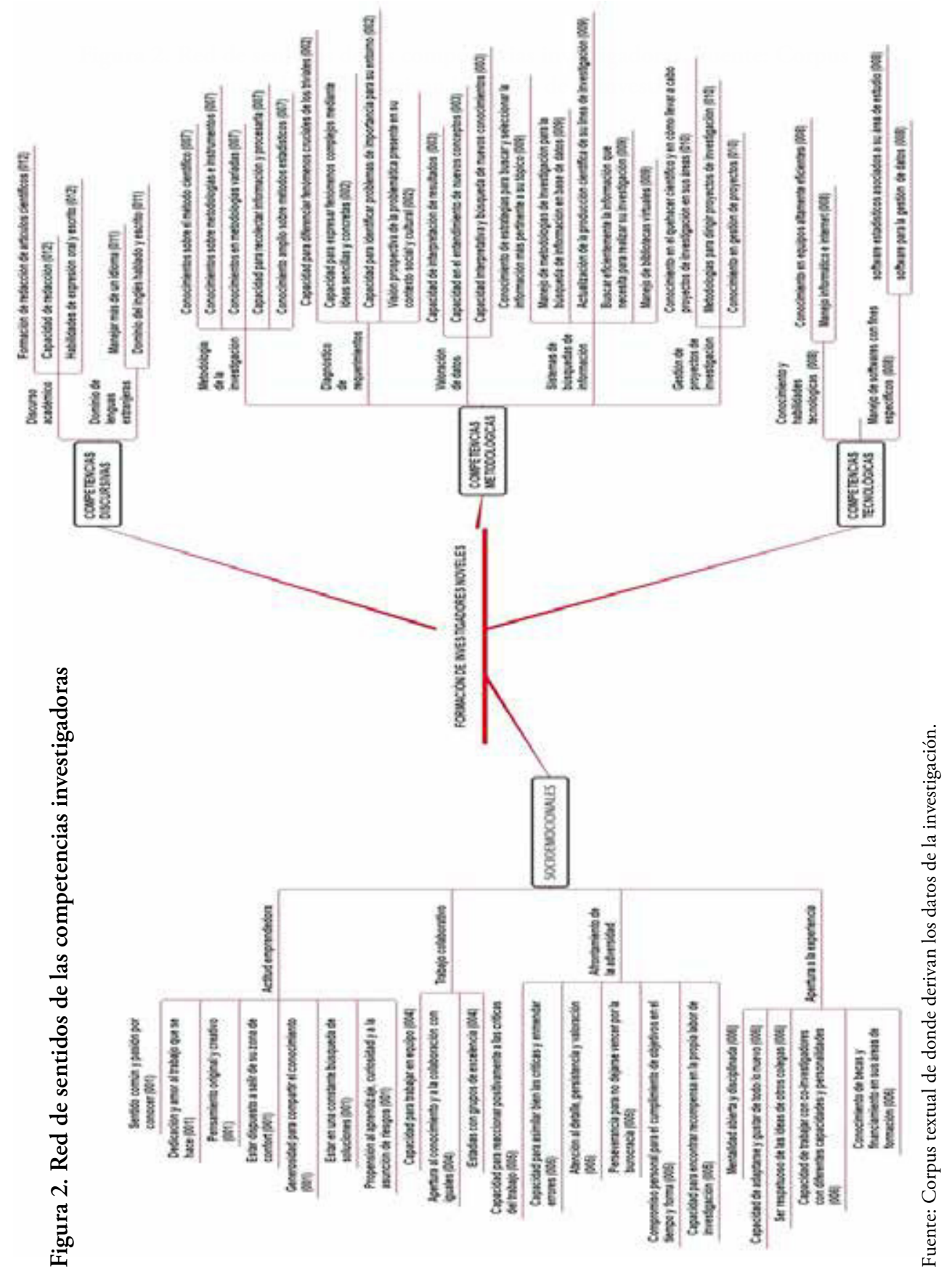


Por último, se presenta la categoría solvencia moral del investigador. Su pertinencia está justificada en lo vulnerable que somos ante la intención de producción y uso del conocimiento, pudiendo direccionarlo hacia la preservación de la humanidad o ponerlo al servicio de las pasiones del poder político-ideológico que reclama espacios más allá de sus fronteras, como sucedió con el proyecto Manhattan y las investigaciones sobre el SIDA. Es en este punto donde tiene sentido la actitud ética del investigador. Su interacción da lugar a la producción de ideas que pueden, o no, tener una dirección constructiva. Este análisis pone la noción del sujeto por encima de los roles que esté llevando a cabo (Zemelman, 1992) e invita a los formadores a potenciar las emociones positivas, la convivencia con el otro, la ética personal en el ejercicio de la autonomía individual para elegir y decidir y, no menos importante, la aceptación de las diferencias como insumo para poder adquirir los saberes específicos bajo el enfoque aprender haciendo (Flores, 2011; Fonseca de Rocca y Prieto de Alizo, 2010).

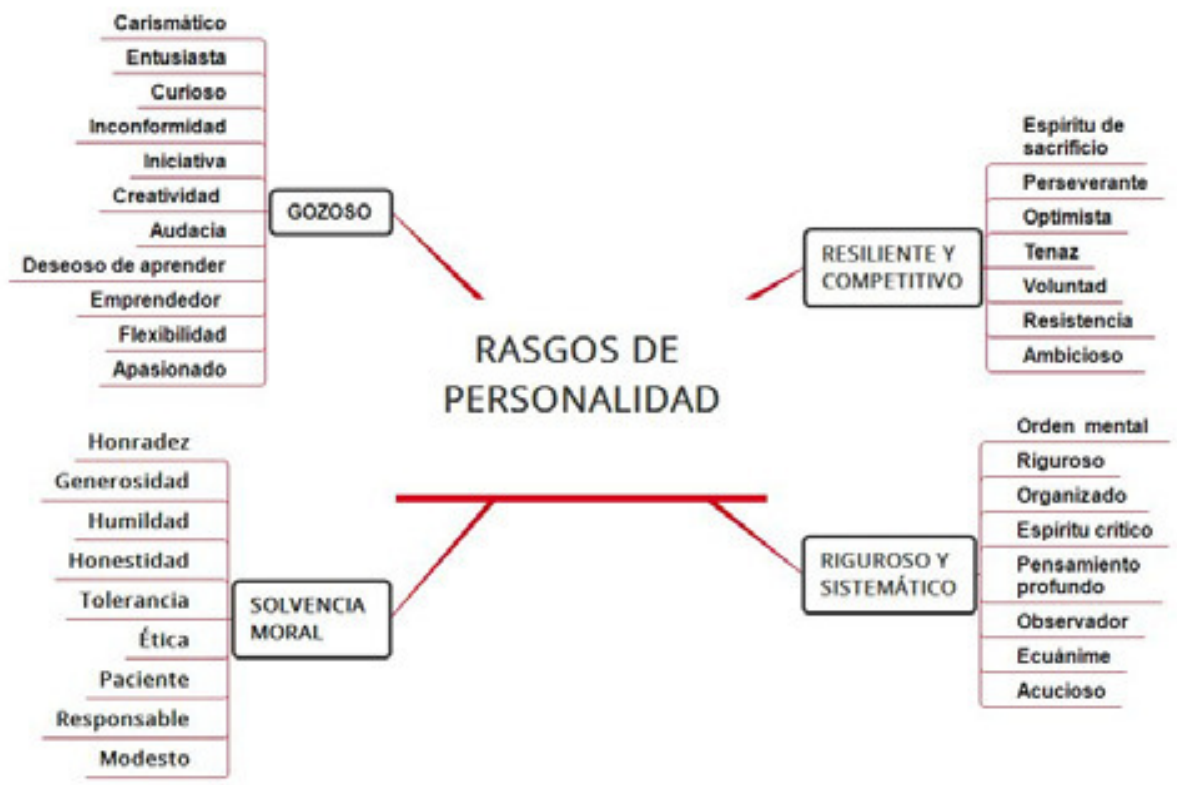

Figura 3. Rasgos de personalidad del investigador novel. Fuente: Corpus textual de donde derivan los datos de la investigación 


\section{Conclusiones}

En este estudio, se demostró que las representaciones de las competencias del investigador novel son construcciones sociocognitivas que median la significación de la experiencia humana, interiorización y los condicionantes de la interacción comunicativa respecto al objeto representado. Esta idea revela que no hay representaciones genéricas ni indiferenciadas. Existen producciones cognitivas con origen y sentido social, de allí que estén sujetas a un proceso de construcción-deconstrucción para garantizar la coexistencia de lineas de sentido. Se expresan mediante el cruce intencionado de capacidades, habilidades, actitudes que condicionan el desempeño del investigador dentro de un contexto sociocognitivo.

Los investigadores consolidados muestran una concepción de la investigación que rescata el carácter emotivo y social de la condición humana. Rompen con la negación de la subjetividad y demuestran que el aplanamiento afectivo al investigar es una utopía, promoviendo la cercanía psicoafectiva entre el que enseńa y aprende para causar mejores resultados en el rendimiento del novel, ya que las emociones afectan el significado del éxito o fracaso de la labor investigativa y gobiernan la tarea de producir conocimiento al punto que ensanchan la capacidad de comprender la realidad que investiga y permiten la experimentación de gozo cuando se mira de frente aquello que se anda buscando, como le sucedió a Sonnebornen en los estudios sobre la conjugación de paramecios (García, 2016; Jiménez y Duarte, 2013; Klotz y Neubaum, 2016; Ruiz, 2016)

Junto a las competencias emocionales, se encontraron las metodológicasdiscursiva-tecnológicas. Este trío engloba el elemento tangible de la acción investigadora. Pone al descubierto los elementos cognitivos y procedimentales que se deben manejar para alcanzar la producción de saberes científicos. Aquí quedó demostrado que existe apertura hacia la pluralidad metódica, al poner de manifiesto que no hay «un método» para investigar. La producción de conocimiento científico reconoce «los métodos» y busca establecer correspondencia entre estos y el objeto de estudio. En consecuencia, las reglas metodológicas dogmáticas terminan siendo una pasión individual de quienes administran la metodología. La idea es tejer puentes transhistóricos y transindividuales que nos hagan funcionar como miembros de una macro red de sentidos y significados que, en definitiva, buscan que el mundo se haga más inteligible.

En esta investigación solo leímos el comportamiento discursivo del investigador experto, sin embargo, es necesario continuar la línea de investigación indagando los sentidos que la formación de investigadores tiene en los noveles. Hace falta saber cuál es el sentido que estas competencias tienen en ellos, a fin 
de definir programas formativos que promuevan significados que trasciendan lo curricular y hagan de la investigación un estilo de vida.

\section{REFERENCIAS BIBLIOGRÁFICAS}

Abric, J. C. (2001). Prácticas sociales y representaciones. México: Ediciones Coyoacán.

Banchs, M. (2000). Aproximaciones procesuales y estructurales al estudio de las representaciones sociales. Paper on Social Representations, 9(3),1-15.

Banchs, M. (2001). Jugando con las ideas en torno a las representaciones sociales desde Venezuela. Fermentun, 11(30), 11-32.

Banchs, M. (2007). Entre la ciencia y el sentido común: representaciones sociales y salud. En Rodriguez Salazar, T. y García Curiel, M. D. Representaciones sociales. Teoría e investigación (pp. 219-254). Guadalajara, México: CUCSH-UDG.

Bayón, M. (2015). La construcción del otro y el discurso de la pobreza Narrativas y experiencias desde la periferia de la ciudad de México. Revista Mexicana de Ciencias Politicas y Sociales, 60(223), 357-376. https://doi.org/10.1016/ S0185-1918(15)72141-0

Bécue, M., Lebart, L. y Rajadell, N. (1992). El análisis estadístico de datos textuales. La lectura según los escolares de enseñanza primaria. Anuario de Psicología, (55), 7-22.

Bisquerra, R. (2005). La educación emocional en la formación del profesorado. Revista interuniversitaria de formación del profesorado, 19(3), 95-114.

Bisquerra, R. y Pérez, N. (2007). Las competencias emocionales. Educación XXI(10), 61-82. https://doi.org/10.5944/educxx1.1.10.297

Blumer, H. (1969). Symbolic interactionism. Perspective and method. Englewood Cliffs, NJ: Prentice-Hall.

Bonilla-Castro, E. y Rodríguez, P. (2005). Más allá del dilema de los métodos: La investigación en ciencias sociales. Bogotá: Editorial Norma.

Caro, M. C. y Sicilia, M. (2014). Las emociones y la resistencia al cambio de las actitudes. Revista Española de Investigación en Marketing ESIC, 18(1), 17-31. https://doi.org/10.1016/S1138-1442(14)60003-6

Cejudo, J. y López-Delgado, M. (2017). Importancia de la inteligencia emocional en la práctica docente: un estudio con maestros. Psicología Educativa, 23, 29-36. https://doi.org/10.1016/j.pse.2016.11.001

Chaui, M. (2006). Cultura e democracia: o discurso competente e outras falas. São Paulo: Cortez. 
Creswell, J. (2014). Qualitative, quantitative, and mixed methods approaches. Thousand Oaks, CA: Sage.

Creswell, J. y Zhang, W. (2009). The Application of Mixed Methods Designs to Trauma Research. Journal of Traumatic Stress, 22(6), 612-621. https://doi. org/10.1002/jts.20479

Criollo, M., Romero, M. y Fontaines-Ruiz, T. (2017). Autoeficacia para el aprendizaje de la investigación en estudiantes universitarios. Psicología Educativa, 23(1), 63-72. https://doi.org/10.1016/j.pse.2016.09.002

Diaz, R. y Gómez, M. (2005). Representaciones del ambiente y organización social del espacio. Caso: comunidad del sector "Chino Julio» del municipio Maracaibo del Estado Zulia, Venezuela. Espacio Abierto. Cuaderno Venezolano de Sociología, 14(4), 609-629.

Dietz, G. (2014). La formación de investigadores en educación y la producción de conocimiento: El caso del Doctorado en Educación de la UATx. Perfiles Educativos, 36(146), 202-205. https://doi.org/10.1016/ S0185-2698(14)70136-9

Doise, W., Spini, D. y Clémence, A. (1999). Human rights studied as social representations in a cross-national context. European Journal of Social Psychology, 29, 1-29. https://doi.org/10.1002/(SICI)10990992(1999902)29:1\%3C1 ::AIDEJSP909\%3E3.0CO;2\#

Durkheim, E. (2000). Representaciones individuales y representaciones colectivas, en sociología y filosofía. Madrid, España: Miño y Dávila.

Engler, B. (2013). Personality Theories. An introduction (ninth edition ed.). Belmont, CA: Wadsworth Cengage Learning.

Fairclough, N. (2008). A dialectical-relation approach to critical discourse analysis in social research. En R. Wodak y M. Meyer. (Ed.), Methods in critical discourses analysis. Londres: Sage. https://doi.org/10.11606/issn.2236-4242. v25I2P307-329

Fairclough, N. (2012). Análise crítica do discurso como método em pesquisa social científica. Linha d'Água, 2(25), 307-329.

Feyerabend, P. (2008). Adiós a la razón. Madrid: Tecnos.

Feyerabend, P. (1987). Contra el método: Esquema de una teoría anarquista del conocimiento. Barcelona: Ariel.

Flament, C. (2001). Estructura, dinámica y transformación de las representaciones sociales. En J. Abric (Ed.), Prácticas sociales y representaciones (pp. 33-52). México: Ediciones Coyoacán.

Flores, M. (2011). Las emociones en la filosofía de la ciencia. Astrolabio. Revista Internacional de Filosofía (12), 37-46. 
Fonseca de Rocca, R. y Prieto de Alizo, L. (2010). Las emociones en el investigador humanista. Omnia, 16(2), 132-149.

Fontaines-Ruiz, T. (2012). Metodología de la investigación. Pasos para realizar el proyecto de investigación. Caracas, Venezuela: Júpiter.

Fontaines-Ruiz,T. (2012). Factores condicionantes dela productividad del científico venezolano: una mirada desde el discurso de los investigadores. Hallazgos, 9(18), 15-32. https://doi.org/10.15332/s1794-3841.2012.0018.01

Fontaines, T. y Jiménez, Í. (2016). Introyección de saberes metodológicos transpuestos en los trabajos de titulación de grado. Estudios Pedagógicos XLII, 2, 113-127. Recuperado de http://mingaonline.uach.cl/pdf/estped/v42n2/ art07.pdf

Foucault, M. (2001). Los anormales. Curso del Collège de France (1974-1975). (V. Marchetti, A. Salomoni, Eds., y H. Pons, Trad.). Madrid: Akal.

Foucault, M. (2005). Vigilar y castigar. Nacimiento de la prisión (34ª ed.). México: Siglo XXI editores.

García, A. (2016). La emoción del descubrimiento científico. La Lección Magistral Andrés Laguna 2015. Educación Médica, 17(2), 38-44. https:// doi.org/10.1016/j.edumed.2016.02.012

Giménez, N., Pedrazas, D., Medina, E. y Dalmau, D. (2009). Formación en investigación: autopercepción de los profesionales sobre sus necesidades. Medicina Clínica, 132, 112-117. https://doi.org/10.1016/j. medcli.2008.09.001

Gimeno, J. (2008). Diez tesis sobre la aparente utilidad de las competencias en educación. En J. Gimeno Sacristan (Ed.), Educar por competencias ¿qué hay de nuevo? (pp. 15-58). Madrid: Morata.

Gotuzzo, E., González, E. y Verdonck, K. (2010). Formación de investigadores en el contexto de proyectos colaborativos: experiencias en el instituto de medicina tropical "Alexander Von Humboldt», Universidad Peruana Cayetano Heredia. Revista Peruana de Medicina Experimental y Salud Pública, 27(3), 419-427. https://doi.org/10.1590/S1726-46342010000300015

Gutiérrez, S. y Campos, D. (2010). El escándalo político. Su estudio desde las representaciones sociales. Anuario de investigación UAM-Xochimilco, 155-183.

Gutiérrez, S. y Cuevas, Y. (2012). Representaciones sociales de Enrique Peña Nieto, candidato a la presidencia de México 2012-2018, en la prensa escrita. Cultura y representaciones sociales, 7(13), 63-95.

Jiménez, V. y Duarte, S. (2013). Características del perfil de los investigadores categorizados por el Consejo Nacional de Ciencia y Tecnología del Paraguay. Revista Internacional de Investigaciones en Ciencias Sociales, 9(2), 221-234. 
Jodelet, D. (2007). Imbricaciones entre representaciones sociales e intervención. En T. Rodríguez Salazar y M. D. García Curiel. (Ed.), Representaciones sociales: teoría e investigación (pp. 191-218). Guadalajara, México: CUCSH-UDG.

Klotz, A. y Neubaum, D. (2016). Research on the Dark Side of Personality Traits in Entrepreneurship: Observations from an Organizational Behavior Perspective. Entrepreneurship Theory and Practice, 40(1), 7-17. https://doi. org/10.1111/etap.12214

Kostrova, O. y Kulinich, M. (2015). Text Genre «Academic Writing»: Intercultural View. Procedia - Social and Behavioral Sciences, 206, 85-89. https://doi. org/10.1016/j.sbspro.2015.10.032

Lakatos, I. (2011). Historia de la Ciencia y sus reconstrucciones racionales. (D. Ribes Nicolás, Trad.) Madrid: Tecnos.

Lancia, F. (2004). Tool for text analysis. Introduction to the use of T-lab. Milán: Franco Angeli.

Lebart, L. y Salem, A. (1994). Statistique Textuelle. París: Dunod.

Lévy-Leboyer, C. (1997). Gestión de las competencias. Barcelona: Gestión 2000.

Martínez-Lorca, M., Zabala-Baños, M. C. y Aguado, R. (2017). Enseñando en la universidad a gestionar las emociones y a establecer el vínculo terapéutico con el paciente. Enfermería Clínica. https://doi.org/10.1016/j. enfcli.2016.11.002

McClelland, D. (1973). Testing for competence rather than for «inteligence». American Psychologist, (14). https://doi.org/10.1037/h0034092

Merton, R. (1977). The sociology of Sciencie. Theoretical and empirical investigations. Chicago: The University Chicago Press.

Mora, M. (2002). La teoría de las representaciones sociales de Serge Moscovici. Atenea Digital (2).

Moreno-Casbas, T., Fuentelsaz-Gallego, C., González-María, E. y Gil de Miguel, Á. (2010). Barreras para la utilización de la investigación. Estudio descriptivo en profesionales de enfermería de la práctica clínica y en investigadores activos. Enfermería Clínica, 20(3), 153-164. https://doi.org/10.1016/j. enfcli.2010.01.005

Moscovici, S. (1979). El psicoanálisis, su imagen y su público. (N. Finetti, Trad.) Buenos Aires, Argentina: Huemul.

Netzahualcoyoti-Netzahual, M.-A. (2015). Las representaciones sociales de los docentes sobre la reforma integral de la educación básica. Revista iberoamericana de educación superior, VI(15), 3-25. https://doi.org/10.1016/ S2007-2872(15)30001-9 
Oriol-Granado, X., Mendoza-Lira, M., Covarrubias-Apablaza, C.-G. y MolinaLópez, V.-M. (2017). Emociones positivas, apoyo a la autonomía y rendimiento de estudiantes universitarios: el papel mediador del compromiso académico y la autoeficacia. Revista de Psicodidáctica, 22(1), 45-53. https:// doi.org/10.1016/S1136-1034(17)30043-6

Parkinson, J., Demecheleer, M. y Mackay, J. (2017). Writing like a builder: Acquiring a professional genre in a pedagogical setting. English for Specific Purposes, 46, 29-44. https://doi.org/10.1016/j.esp.2016.12.003

Rico, D., Alzate, M. y Sabucedo, J. M. (2017). El papel de la identidad, la eficacia y las emociones positivas en las acciones colectivas de resistencia pacífica en contextos violentos. Revista Latinoamericana de Psicologia, 49(1), 28-35. https://doi.org/10.1016/j.rlp.2015.09.013

Rivas, L. (2004). La formación de investigadores en México. Perfles latinoamericanos, (25), 89-113.

Rodriguez, B., Alvite, M. L. y Olea, I. (2015). La utilización de las revistas electrónicas en la Universidad de León (España): hábitos de consumo y satisfacción de los investigadores. Investigación Bibliotecológica: Archivonomía, Bibliotecología e Información, 29(66), 17-55. http://dx.doi.org/10.1016/j. ibbai.2016.02.024

Ruiz, S. (2016). La subjetividad del investigador en terreno: Sistematización de una experienciareflexivadeinmersiónetnográfica.Psicoperspectivas, 15(1),42-52. https://doi.org/10.5027/psicoperspectivas-Vol15-Issue1-fulltext-714

Salgado, P. M., Ortiz, D. A. C. y Rogel, R. M. N. (2016). La interdisciplinariedad económico-administrativa en la conformación de una comunidad científica y la formación de investigadores. Revista de La Educación Superior, 45(177), 43-65. https://doi.org/10.1016/j.resu.2015.12.011

Tobon, S., Rial Sanchez, A., Carretero, M. y Garcia, J. (2006). Competencias, calidad y educación superior. Colombia: Alma Mater Magisterio.

Tonso, K. L. (2006). Student engineers an engineer identity: Campus engineer identities as figured world. Cultural Studies of Science Education, 273-307. https://doi.org/10.1007/s11422-005-9009-2

Torrecilla, M., Cartagena, E., Crespo, A. P., Oyarzabal, M. y Pérez, C. I. (2017). Formación en investigación para tutores y tutoras de una Unidad Docente Multiprofesional provincial de Atención Familiar y Comunitaria. Educación Médica. https://doi.org/10.1016/j.edumed.2017.03.007

Torres-Moreno, J.M., Molina, A. y Sierra, G. (2010). La energía textual como medida de distancia en agrupamiento de definiciones. En S. Bolasco, I. Chiari, y L. Giruliano, Statistical analysis of textual data. Proceedings of 10th International Conference Journées d'Analyse statistique des données textuelles 
9-11 de Junio de 2010-Sapienza University of Rome (pp. 215-226). Roma: Sapienza University of Rome.

Viard, D., Netillard, C., Cheraitiaa, E., Barthoda, V., Choffela, J., Tartarya, D. y Tissot, E. (2016). Éducation thérapeutique en psychiatrie: représentations des soignants, des patients et des familles. L'Encéphale, 42(1), 4-13. https://doi.org/10.1016/j.encep.2015.08.003

Wundt, W. (1926). Elementos de psicología de los pueblos. Bosquejo de una historia de la evolución psicológica de la humanidad. Madrid: Alta Fulla.

Zare, J. y Keivanloo-Shahrestanaki, Z. (2017). Genre awareness and academic lecture comprehension: The impact of teaching importance markers. Journal of English for Academic Purposes, 27, 31-41. https://doi.org/10.1016/j. jeap.2017.03.001

Zemelman, H. (1992). Los horizontes de la razón. Barcelona: Antropos.

Zhang, L., Kong, M. y Li, Z. (2017). Emotion regulation difficulties and moral judgment in different domains: The mediation of emotional valence and arousal. Personality and Individual Differences, 109, 56-60. https://doi. org/10.1016/j.paid.2016.12.049 